\title{
COMENTARIOS SOBRE LA INVESTIGACIÓN CIENTÍFICA
}

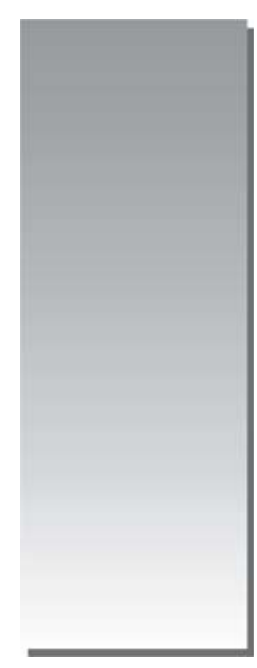

\author{
Eulogio Hurtado Dianderas * \\ E-mail: eulogio1952@hotmail.com
}

\begin{abstract}
RESUMEN
En el presente artículo escribimos algunas reflexiones sobre la investigación científica como temática, destacando su conceptualización y lo relacionado con la creatividad e innovación, con la finalidad de motivar a los lectores al estudio de la investigación científica.

El presente texto busca definir la red compleja de la investigación científica en concatenación con el conocimiento, la creatividad y el mundo académico; en el desarrollo científico, en !a tecnología y como disciplina. Para ello tomamos algunas reflexiones de los grandes pensadores en esta área llegando a la conclusión que la investigación científica es un proceso que no sigue un camino trazado de antemano en todos sus detalles, o una vereda única que debe de recorrerse a ciegas o mecánicamente, y cuya finalidad es obtener la verdad al final de la senda.

El método es un hilo conductor que orienta el trabajo científico; es por ello que se afirma que la investigación es un proceso que se construye a medida que avanzamos, en donde existen veredas suficientes dejadas por otros científicos para no perderse y que se encuentran en peligro de ser cubiertas por la maleza de la complejidad social de la vida.

Quiero finalmente expresar que el Método Científico no es ni más ni menos que la manera de hacer buena ciencia, natural o social, pura o aplicada, formal o fáctica y que puede adaptarse a campos que antes no eran científicos porque se caracterizan, al igual que la ciencia, por la búsqueda de pautas generales.

Palabras Clave: Investigación científica, paradigma, sistemas, emergencias, control, comunicación, holístico, transdisciplinidad, cosmológico, ecológico, hermenéutico, complejidad, temática, dialéctica, indagación, tecnología.
\end{abstract}

\begin{abstract}
In the current article we wrote some reflections on scientific research, like thematic, emphasizing his conceptualization and relatioship to creativity and innovation, with the purpose of motivating the readers into the studying of scientific research.

The current text looks for to define the complex network of scientific research concatening knowledge, creativity an academic world; in scientific development, in technology and as a discipline. Which is why we took some reflections from the great minds in this area, arriving to the conclusion that the scientific
\end{abstract}

* Profesor de Pre y Post Grado de la Facultad de Ciencias Administrativas (UNMSM). Profesor de Post Grado de la Facultad de Ingeniería Industrial y de la Facultad de Ingeniería Geológica Minera, Metalúrgica y Geográfica (UNMSM). 
research is a process that does not follow a way traced up before in all its details, or a unique path that must be crossed on blind or mechanically, and whose purpose is to obtain the truth at the end of the path.

The method is a wire that guides the scientific work; that is why it is affirmed that research is a process that is constructed as we go through, where there are sufficient paths left by other scientists for not to lose oneself an that are in danger to be covered by the weeds of social life complexity.

Finally, I want to express that scientific method is neither the more nor the less than the way to make good science, natural or social, pure or applied, formal or factic and that can be adapted to fields that before were not scientific because they are characterized, like science, for the search of general guidelines.

Key words: scientific research, paradigm, systems, emergencies, control, communication, holistic, transdisciplinity, cosmological, ecologic, hermeneutic, complexity, thematic, dialectic, indagation, technology.

\section{INTRODUCCIÓN}

Meditar, reflexionar sobre la Investigación Científica como temática es una tarea difícil, pero trataremos de analizar el papel de la investigación desde diferentes perspectivas como la realidad académica, su desarrollo científico, en la tecnología, en la innovación tecnológica y en su desarrollo como disciplina.

La investigación en el mundo académico, es decir en la universidad, se realiza con la finalidad de aportar a la construcción de conocimiento del país; para generar actitudes científicas; para contribuir a que la ciencia y la tecnología formen parte de la estructura social como elementos indispensables para el mejoramiento de !a calidad de vida; y con la finalidad de contribuir en la consolidación del sistema educativo.

La investigación desde el punto de vista de la tecnología o como temática de la ingeniería, es de carácter teórico y práctico, fenómenos que no pueden presentarse de manera aislada e inconexa sino como un todo, formando una malla compleja en la realidad, con la participación activa del Hombre.

La investigación se puede realizar de diferentes maneras, como: metodología de estudio: de investigación bibliográfica y del trabajo científico, como metodología de investigación científica y como metodología de ingeniería.

\section{LA INVESTIGACIÓN CIENTÍFICA SIGUE UN CAMINO TRAZADO}

En primera instancia debemos presentar una definición de investigación científica, la cuál es considerada como un proceso que tiene como finalidad lograr un conocimiento objetivo, es decir verdadero sobre determinados aspectos de la realidad, con la finalidad de usarlo como camino de la práctica transformadora del hombre.

Es necesario tener en consideración que en el proceso de transformación del mundo se encuentra presente lo que llamarnos dialéctica materialista, que debe considerarse como una característica intrínseca de los fenómenos y procesos de la naturaleza, la sociedad y el pensamiento, la cual estará presente en toda investigación.

Debernos destacar que, en la práctica, la investigación ha demostrado ser un proceso ligado a una compleja malla de realidad, lo que permite decir que la investigación no sigue un modelo o esquema rígido ni es una serie de etapas ligadas mecánicamente. Si concebimos a la investigación como un proceso concatenado de etapas significaría que la naturaleza estaría muerta, paralizada, sin cambios, pues en realidad sucede todo lo contrario: la naturaleza se encuentra en un permanente cambio y desarrollo, adoptando múltiples formas y en una constante relación en el devenir histórico que no tiene punto final.

Quiero decir que en realidad el método de investigación no puede abarcar todas las particularidades o manifestaciones concretas de cualquier proceso de la realidad que se estudia, ya que ésta desbordará -como un río caudaloso e incontenible- aquellas señales teóricas metodológicas rígidas consideradas como muchos como verdades definitivas que deben de seguirse al pie de la letra para llevar a cabo nuestro trabajo científico. 
En realidad el método de investigación, los principios metodológicos, se refieren a los aspectos más generales que orientan los procesos concretos de investigación, pues en la práctica investigativa los lineamientos metodológicos resultan insuficientes para alcanzar la verdad científica. Se debe de recurrir a métodos específicos, a técnicas e instrumentos adecuados y precisos para recopilar y analizar la información empírica que la teoría utilizada y las hipótesis plantean, y señalan como relevantes para la formulación de conocimiento científico.

Teniendo en mente estas reflexiones ponemos decir que la investigación contribuye a afinar, a enriquecer el método científico, en un proceso que no tiene fin, destacando que entre el nivel singular y general existen diversos niveles intermedios; en otras palabras, hay aspectos del método de investigación más generales o más concretos que otros, y su forma de vincularse dependerá de lo que se investiga, cómo se investiga y quién lo investiga.

También es necesario resaltar que la comprensión y el manejo de los elementos teóricos, metodológicos y técnicos no son llevados a cabo de igual manera por las personas que indagan sobre el mismo tema; de igual manera, la realidad que se investiga no es siempre la misma, aún cuando a veces tenernos la impresión de que no cambia como consecuencia de que se observan solo momentos de su desarrollo pues la mente tiende a paralizarnos y la historia parece repetirse, pese a que no existen dos fenómenos iguales dentro de un mismo objeto o proceso.

Debemos tener presente que la realidad es y no es al mismo tiempo; es decir, existe y deja de ser, cambia constantemente impulsada por la contradicción de sus elementos antagónicos, si no recordemos lo que dijera Heráclito, padre de la dialéctica: «No podemos bañarnos dos veces en las mismas aguas, pues aún cuando el río permanece las aguas ya no son las mismas».

En realidad, el investigador se encuentra situado en un momento histórico del fenómeno que estudia, lo cual no significa que sea definitivo, absoluto, sino que es solo un momento del desarrollo de la realidad pues por una necesidad de abstracción tiene que paralizar en la mente determinados aspectos y relaciones de los procesos a fin de profundizar en su análisis. Es de- cir, es como si la realidad fuese una película y la detenemos en cierto punto para observar detalles que resulta importante indagar de acuerdo con nuestra hipótesis y objetivos de la investigación, aún cuando sabemos que la realidad es un proceso y como tal continúa su movimiento sin detenerse jamás, adoptando nuevos aspectos y relaciones entre los fenómenos.

Es preciso destacar que, dentro de esos cambios, existen elementos cuya variabilidad es menor o que requieren mayor tiempo para sufrir alteraciones cualitativas; esa invariabilidad dentro de la variabilidad es lo que interesa a la ciencia, con la finalidad de convertir en leyes científicas las leyes naturales que gobiernan el mundo real y social, para poder explicar y predecir los fenómenos objeto de preocupación.

Finalmente, de las reflexiones hasta aquí expuestas, podemos decir que la investigación científica es un proceso que no sigue un camino trazado de antemano en todos sus detalles, o que es una vereda única que debe de recorrerse a ciegas y mecánicamente, con la finalidad de obtener al final de la senda, la verdad.

En la investigación no caben las concepciones rígidas que limitan el pensamiento y la acción; es decir, se requiere un espíritu dialéctico que conjugue la preparación formal en el campo de la teoría, la metodología y la técnica, con las experiencia directas e indirectas, así como la imaginación creativa e innovadora que es una de las principales riquezas de la humanidad.

\section{LA INVESTIGACIÓN Y EL MÉTODO}

Al respecto, diremos que una diversidad de autores afirma que el método es el camino que se sigue en la investigación, pero en realidad nos preguntamos ¿cuál es ese camino?, ¿ese camino está trazado de una vez y para siempre y basta seguirlo para alcanzar la verdad científica? Pues tal señalamiento sólo permite mostrar que el método es un hilo conductor que orienta el trabajo científico; es por ello que se afirma que la investigación es un proceso que se construye a medida que avanzamos, en donde existen veredas dejadas por otros científicos para no perderse y que se encuentran en peligro de ser cubiertas por la maleza de la complejidad social de la vida.

Solo como para refrescar la mente podemos repetirlo que decía Antonio Machado que en la 
investigación «no hay camino, se hace camino al andar», camino que no está trazado al azar o de manera caprichosa. En la investigación debemos seguir las huellas de otros investigadores quienes han dejado metodologías en el camino de la investigación, en los cuales observamos desviaciones y que están plagados de una cantidad abrumadora de datos, conceptos y lagunas teóricas. En realidad, la forma como recorramos la práctica de la investigación dependerá de aspectos objetivos y subjetivos, internos y externos al sujeto y el objeto de su estudio.

Las reflexiones anteriores no tienen el norte de negar al método como plantean muchos autores, puesto que la investigación se plantea y desarrolla en base a los lineamientos metodológicos existentes y de acuerdo con la teoría e hipótesis que guían la apropiación teórica de la realidad concreta a través de contactos directos y permanente, lo que conforma la práctica científica.

Finalmente podemos decir que el diseño de la investigación no sustituye a la investigación misma, pues es parte integral de ella, y sirve para guiar nuestro trabajo por sendas fecundas para la ciencia.

\section{LA INVESTIGACIÓN CIENTÍFICA Y LA CIENCIA}

En esta oportunidad tenemos que citar a los dos más conspicuos metodólogos, M. Bunge y Kopnin, quienes consideran a la ciencia como el sistema del conocimiento y leyes, y como investigación a la manera como se observan estos dos elementos básicos que definen a la ciencia, y que se encuentran fuertemente interrelacionados formando la red compleja de la ciencia.

Sin embargo el conocimiento científico es la emergencia o sinergia de la investigación y, por consecuencia, la investigación científica es el nivel de desarrollo de su método y que es resultado de la sinergia del avance del conocimiento científico.

Considerando estas reflexiones podemos afirmar que la ciencia es, por un lado, conocimiento, y por otro, investigación, formando éstas dos caras de la misma medalla, contradictorias pero que se encuentran fuertemente interrelacionadas; podríamos decir que formando un isomorfismo compacto.

Quisiera tomar las palabras de M. Bunge al respecto de este ítem: «Tenemos que distinguir en la ciencia entre el trabajo - investigación y su producto final». Observamos que esta expresión parecería indicar que el ciclo se cierra en el producto final, fenómeno que no es cierto. El producto final es resultado de la investigación, pero al mismo tiempo es también la base para iniciar un nuevo ciclo, es el inicio de un nuevo ciclo de búsqueda de nuevos conocimientos. Para Bunge, en realidad, cada ciclo representa un eslabón de una cadena inmensa que va creciendo hacia el infinito ${ }^{1}$.

También quiero realizar algunas reflexiones sobre Kopnin, gran personaje de la investigación científica. Para Kopnin las articulaciones entre el conocimiento y la investigación son más precisas. El conocimiento científico refleja las leyes fundamentales que rigen el desarrollo de los fenómenos, leyes que constituyen el cordón umbilical del nuevo conocimiento científico, pero que en realidad se transforman en las palancas metodológicas que permitirán aprender nuevos conocimientos. En otras palabras, lo reflejado se convierte en método de investigación.

Parafraseando a Kopnin «Las leyes objetivas, transplantadas y transformadas en la mente humana, se convierten en método del conocimiento» ${ }^{2}$. Observemos que no solo se destaca la relación conocimiento y método sino que va más allá: el uno se transforma en el otro.

\section{LA INVESTIGACIÓN CIENTÍFICA Y BUNGE}

Bunge es uno de los más versados y cotizados estudiosos que tiene la Metodología de la Investigación Científica (MIC). Afirma que la MIC "Consiste constantemente en tratar problemas» con el método y el objeto de la ciencia. La Investigación Científica (IC) encuentra, plantea, resuelve y genera nuevos problemas, y permanentemente está tratando con problemas. Pero debemos destacar que el tratamiento no es realizado de cualquier manera si no que sigue los lineamientos del método científico, y, sólo de esta manera es posible llegar a encontrar las leyes científicas que son el objetivo de la ciencia.

Cuando M. Bunge define MIC encontramos básicamente tres ingredientes fundamentales: el problema, el método y la ley, que son los mismos que forman el esquema de su definición de IC: problema - método - ley.

- El problema. Pregunta o fenómeno a resolver, es el elemento generador, o como Bunge dice, el muelle de la investigación. 
- El Método. El procedimiento, los pasos para tratar el problema o fenómeno que se quiere resolver o estudiar; es decir, es el ente de trabajo.

- La Ley. Nuevo conocimiento obtenido o la sinergia de la interrelación compacta entre el problema y el método.

Quiero destacar que si realizamos una lectura unilateral a M. Bunge podemos identificar incorrectamente los siguientes fenómenos: investigación igual al problema; investigación igual a método científico; finalmente, investigación igual a leyes, pues Bunge afirma que la «Investigación consiste constantemente en tratar problemas» o que «los problemas son los muelles que impulsa la actividad científica» o que "son la fuente de la ciencia» ${ }^{3}$, lo que puede dar lugar a que se exclusivice esta idea en la definición de la investigación científica, dejando de lado los otros elementos que forman su terna fundamental.

De igual manera si se le reduce a la idea de que «donde no hay método científico no hay ciencia», podemos quizás confundir la investigación científica con el método científico, mucho más aún si concebimos el método científico como un conjunto de pasos que incluye el problema, la hipótesis y la contrastación de hipótesis hasta llegar al nuevo cuerpo del conocimiento.

De igual modo sería incorrecto identificar investigación con leyes a partir de la consideración siguiente: «... la investigación científica es, dicho brevemente, la búsqueda de la estructura» o de las leyes. En realidad, la investigación científica para Bunge, es todo esto pero formando una complejidad, es decir una compleja malla en la cual el Método Científico desempeña un papel central, porque estudia, analiza y constituye la teoría de la investigación.

Veamos la teoría que tiene Bunge sobre el Método Científico (MC), cuyo estudio nos conducirá a encontrar los fundamentos que subyacen en su propuesta sobre la investigación científica.

El Método Científico o Método General de la Ciencia como lo llama Bunge «...es un procedimiento, es la estrategia de la investigación científca que afecta todo el ciclo completo de la investigación y que es independiente del tema en estudio». El ciclo está constituido por un conjunto de pasos que, más allá del tema concreto de estudio, recorre toda investigación científica.
Entre los elementos que se destacan en la manera de reflexionar de Bunge, tenemos:

- Estrategia, que señala los pasos lógicos generales que hay que dar en la investigación.

- Validez de la estrategia para el conjunto de las ciencias.

Pero en realidad nos resume algo que nos parece central y que puede percibirse con mucha claridad en el texto que citamos, así como en su epistemología: la manera general de dar el conjunto de pasos para llegar a descubrir las leyes. Aún así considerando que existe en Bunge una lógica que le da sentido a esos pasos generales, hay un gran vacío, pues carece de una teoría global de la realidad objetiva, del desarrollo de los fenómenos, donde se puedan encuadrar esos pasos generales y tengan un sentido general esencial y no aparente, en superficie, como ocurre realmente; pues la teoría señala el norte, proporciona el cordón umbilical y articula los pasos dentro de una totalidad, reflejada en otras totalidades, que es la realidad objetiva.

Quiero reflexionar aún más, pues Bunge nos habla y teoriza sobre el Método General de la Ciencia o Método Científico creo yo, sin tener un enfoque, sin tener una teoría general de la realidad. Para Bunge, su Método Científico trasciende lo particular, lo especial, y es forzoso que haya sido el resultado de una comprensión de la realidad objetiva en general, lo cual no es posible sin un planteamiento teórico de la misma. Nos parece que ésta es la razón por lo cual Bunge define el Método Científico tomando como eje de los pasos el procedimiento que se sigue en la investigación, pero sin precisar el sentido esencial del conjunto de los pasos.

Es cierto que nos dice cómo hay que dar los pasos, que tienen una lógica, pero este cómo y esta lógica tiene un contenido que refleja parcialmente e unilateralmente la realidad general y no sus leyes.

En realidad el modo de dar esos pasos generales, su lógica, aparecen independientes de las leyes generales que presiden al desarrollo de la realidad objetiva. Es por eso que en nuestra opinión, Bunge no puede avanzar hacia la articulación de sus pasos, su lógica, con las leyes de la realidad general porque carece de una teoría del mundo objetivo. 
Según Bunge, los pasos del Método Científico que toda investigación científica debe seguir, son:

1. Descubrimiento del problema o laguna en un conjunto de conocimientos.

2. Planteo preciso del problema.

3. Búsqueda de conocimientos o instrumentos relevantes al problema.

4. Tentativa de solución del problema con ayuda de medios identificados.

5. Invención de una nueva idea (hipótesis, teorías o técnicas) o producción de nuevos datos empíricos que prometen resolver el problema.

6. Obtención de una solución (exacta o aproximada) del problema, con ayuda de los instrumentales conceptuales o empíricos disponibles.

7. Investigación de las consecuencias de la solución obtenida.

8. Puesta a prueba (contrastación) de la solución; confrontación de ésta con la totalidad de las teorías y de la información empírica pertinente.

9. Corrección de las hipótesis, teorías, procedimientos o datos empleados en la obtención de la solución correcta.

Estos pasos señalados en su epistemología constituyen el método científico de Bunge sintéticamente. El método científico, en tanto estrategia de la investigación, es concebido como un procedimiento que arranca desde el hallazgo y planteo del problema hasta la solución y creación de una nueva teoría. Nos preguntamos: ćlos pasos citados tienen que ver con la realidad objetiva? $\mathrm{Si}$ esos pasos están ligados a la realidad objetiva ¿reflejarán lo esencial de la realidad?

Reflexionemos sobre esas preguntas. Si los comentarios hechos sobre los pasos se dice que son independientes del tema en análisis, esto no significa, en el concepto del mismo autor, que hayan sido construidos a priori. Por lo contrario, son sistematizaciones que proceden de la realidad objetiva, de la experiencia realizada por el investigador a cargo de descubrir la realidad por lo que tienen validez para cualquier tema y no para uno en especial, aunque es necesario resaltar que esa validez es relativa y depende mucho de cada objeto de estudio.

Con relación a la segunda pregunta, su respuesta es fundamental para comprender las posibilidades y las limitaciones de la concepción del autor y no porque estén articulados ni porque se desprendan de ella, si no que necesariamente reflejarán lo esencial de la realidad.

El concepto sobre el Método Científico de Bunge contiene la forma como éste se va presentando a los investigadores y parte de la forma como se le va problematizando e investigando. Pero estas formas son la naturaleza externa, las cuales tratan de ser resumidas por el autor cuando señala el conjunto de pasos o elementos que forman el Método Científico.

En esta óptica los pasos del método de Bunge resultan ser una descripción solamente, que contiene parte de la realidad más no la esencia de esa realidad, lo cual se traduce en el hecho de que los pasos metodológicos de Bunge operan a nivel externo y descriptivo, y que es solo un nivel del método científico. Operando a ese nivel, nos permiten instrumentarnos para captar parcial, unilateral y superficialmente la realidad.

Finalmente desde nuestro punto de vista, los nueve pasos de Bunge son pasos limitados en ese sentido: por sí solos únicamente ayudan a captar superficialmente la realidad. Pero debemos destacar que sus hallazgos a ese nivel de abstracción de la realidad, tan precisos y exhaustivos cuando se articulan y encausan por una teoría y un método, adquieren una importante potenciación, pues no olvidemos que sus pasos metodológicos no son elucubraciones fantasiosas sino centralmente excelentes sistematizaciones de un aspecto de la realidad, y como tal se debe de señalar su importancia en la búsqueda de la verdad en la investigación científica.

\section{PROCEDIMIENTO CIENTÍFICO}

Lo que me induce a dar ciertas apreciaciones sobre procedimiento científico y método científico es el hecho que generalmente los tratamos como iguales. Pues llamaremos procedimiento científico al conjunto de pasos, normas y reglas que se siguen en una investigación científica; es el acercamiento al conocimiento de los objetos.

Los hombres de ciencia han tenido que dar un conjunto de pasos, normas y reglas de la complejidad nebulosa de los primeros acercamientos a los objetos que se pretendía estudiar, pues la práctica obligaba a los investigadores a sistematizar y ordenar sus procedimientos, llegando a establecer que en la investigación científica se 
tiene el problema, hipótesis, confrontación, comprobación de la hipótesis, y nuevos conocimientos. A su vez, en cada paso, se han establecidos normas y reglas con la finalidad de encausar la investigación.

Podemos decir que existe un procedimiento científico general y procedimientos particulares, donde el primero tiene su campo de acción más amplio y reglas generales a seguir en toda investigación científica.

Los procedimientos particulares se refieren a los pasos normas y reglas a seguir en la investigación de un objeto determinado o en una ciencia determinada, donde ambos procedimientos se encuentran estrechamente relacionados. De esta manera, el planteamiento de un problema es un paso que forma parte del procedimiento general. Cuando M. Bunge nos habla de las condiciones necesarias y suficientes para que un problema pueda considerarse como científico, lo que está haciendo es enunciar un conjunto de normas y reglas generales.

Los procedimientos científicos son abstracciones que los investigadores hacen de la realidad. Reflejan la realidad y la práctica de los investigadores en uno de los niveles de acercamiento a la realidad.

El planteamiento del problema y las hipótesis científicas son formas de pensamiento que reflejan el proceso de acercamiento de los investigadores a la realidad. Sin embargo, debemos decir que estos acercamientos a través de estos procedimientos son muy débiles en la medida en que apuntan a reflejar la envoltura de la realidad o del proceso de investigación. Este es precisamente el punto débil de los procedimientos científicos, sobre todo cuando se les maneja desarticuladamente de una concepción y un método científico. Cuando ocurre esto, el reflejo externo y descriptivo se unilateraliza al extremo y pierde su posibilidad de conducirnos a un buen puerto.

Es preciso mencionar a Rochabrún, en cuanto a la crítica que hace a quienes conciben al método como una serie de procedimientos. Rechaza la metodología que se orienta centralmente en esa dirección: "En ella pueden existir libros de metodología que no dicen explícitamente ni 'a' sobre la teoría ni sobre la realidad y viceversa». Implícitamente contienen muy parcialmente la realidad locuaz que limita y bloquea su capacidad para captarla, esta es la razón de que un profesor o un estudiante puedan aprender todos estos textos pero que no se conviertan en investigadores, puesto que solo tienen un avance formal.

Pero en realidad, si los procedimientos científicos forman parte de una concepción y un método científico, contribuyen con mayor decisión a la investigación de los fenómenos, pues dan un salto y de categorías meramente descriptivas se transforman en categorías teóricas de un mayor nivel, puesto que no solo contienen parte de la realidad sino que se articulan a la realidad general a través de todo el sistema de categorías, leyes, principios y conceptos de la teoría y el Método Científico, dejando de ser meros procedimientos aislados y formando parte del Método Científico.

\section{MÉTODO CIENTÍFICO}

Quiero iniciar este ítem con la siguiente pregunta: ¿qué es el Método Científico? Diremos que es el método que enfoca y capta los fenómenos en su movimiento, desarrollo, sus contradicciones y múltiples relaciones.

Si consideramos la reflexión anterior, podemos decir que solo es posible llegar a encontrar las leyes que gobiernan la vida de los fenómenos si los examinamos en su proceso de desarrollo, tratando de encontrar sus contradicciones que los generan y relacionando los fenómenos.

El enfoque o concepción metodológica científica requiere, para acercarse al fenómeno, de un conjunto de pasos o procedimientos generales y particulares que necesariamente hay que dar para poder encontrar las leyes que rigen los fenómenos; de esta manera, es ineludible plantearse un determinado problema para realizar la investigación. Pero lo sustantivo no se encuentra dado por la convicción de que eso tiene que ser así, si no por la manera o el modo de plantearlo, y este modo de plantearlo estará determinado por el mayor o menor grado de dominio de la concepción metodológica; es decir, por el Método Científico y como tal se debe señalar su importancia en la búsqueda de la verdad en la investigación científica.

- Los procedimientos científicos forman parte de una concepción, y un método científico contribuye con mayor decisión a la investigación de los fenómenos.

- Los procedimientos científicos dan un salto de categorías meramente descriptivas, y se transforman en categorías teóricas de un 
mayor nivel puesto que no solo contienen parte de la realidad sino que se articulan a la realidad general a través de todo el sistema de categorías, leyes, principios y conceptos de la teoría y el Método Científico; dejando de ser meros procedimientos aislados y formando parte del Método Científico.

- Se debe comprender que las técnicas están ligadas al Método Científico; de esta manera el ordenamiento o búsqueda de información estará marcado por él, pues existe una unidad entre el método y las técnicas que, a su vez, forman parte de otra unidad mayor, formando de esta manera la terna teoría, método y técnicas que constituyen la ciencia.

- Todos los profesores tenemos la tarea ineludible que contribuir a desarrollar los hábitos de construir preguntas claras, precisas y pertinentes de la observación, del trabajo sistémico y con propósitos claros de la labor coordinada en equipo, con imaginación y creatividad, propiciando así la actividad investigadora.

- Considerando que la investigación es un oficio, como un trabajo artesanal, entonces la labor del profesor que pretende enseñar a investigar se parece a la tarea del artesano con sus aprendices, es decir, se debe de enseñar haciendo, es decir, investigando más que teorizando.

- La investigación necesita la formación de comunidades o de redes de investigadores que compartan intereses, preferentemente que provengan de diferentes áreas del conocimiento.

- La comunicación de métodos, problemas y resultados es indispensable.

- No existe investigación si ésta no es publicada en seminarios, congresos y en particular libros y revistas.

- La investigación nos da la oportunidad de poner en práctica el trabajo en equipo, en donde se tiene un compañero a quien hacerle una pregunta, discutir ideas y como consecuencia de eso, tener más posibilidades de plantear nuevas interrogantes sobre una problemática.

- La investigación permitirá entender y comprender un concepto en términos de sistema y experimentar la emoción de participar. Permite tener una buena comunicación, obtener experiencia en el manejo de problemas y cambiar metas a la mitad del proyecto.

Consecuentemente el enfoque, la concepción metodológica, es lo esencial del Método Científico, mientras que los pasos o procedimientos son elementos secundarios.

\section{INVESTIGACIÓN CIENTÍFICA Y LAS TÉCNICAS}

En este ítem quisiera también reflexionar sobre las técnicas, pues al tratar sobre la investigación científica señalamos como un elemento importante las técnicas y las acciones concretas que el investigador realiza al buscar la verdad.

En realidad se entiende como técnica al conjunto de medios e instrumentos para recolectar, conservar, clasificar, cuantificar y procesar la información necesaria en toda investigación, formando parte de las normas operativas para elaborar planes y proyectos de la investigación.

Debemos destacar que aparte de la utilización de determinadas técnicas, la investigación requiere de los investigadores una serie de acciones concretas para elaborar, desarrollar e implementar el proyecto de investigación que muchas veces trascienden de lo planificado.

\section{CONCLUSIONES}

1. La investigación enseña que nuestro sistema educativo ha limitado el desarrollo del talento humano al pretender que se aprenda de una sola manera, en un salón de clase y con la asesoría de un profesor como requisitos indispensables. La curiosidad, creatividad, imaginación y el espíritu investigativo no adquieren las dimensiones apropiadas para el presente siglo, y lo mejor que podemos hacer es potenciarlas.

2. El diseño de la investigación no sustituye a la investigación misma, pues es parte de ella y sirve para guiar nuestro trabajo por sendas fecundas para la ciencia.

3. Los nueve pasos de M. Bunge del Método Científico, son pasos limitados en el sentido de que por sí solos únicamente ayudan a captar superficialmente la realidad, pero debemos destacar que sus hallazgos a ese nivel de abstracción de la realidad, tan precisos y exhaustivos, cuando se articulan y encausan por una teoría y un método, adquieren una importante potenciación. No olvidemos que sus pasos 
metodológicos no son elucubraciones fantasiosas sino, centralmente, excelentes sistematizaciones de un aspecto de la realidad.

4. La investigación permite tener un punto de vista de como integrar lo global con lo analítico, puesto que el análisis y la síntesis son dos fases del proceso del pensamiento que necesitan el uno del otro de manera dialéctica.

5. La investigación permite tener una concepción transdisciplinaria y unificada de una problemática; así mismo nos permite hacer una elaboración científica del concepto de totalidad.

6. La investigación permite decir que los docentes no deberíamos contentarnos con solo escondernos en uno de los lados de la balanza, pues debemos seguir la raíz etimológica de investigador, que persigue indicios y huellas o vestigium; como cazador furtivo construye redes sobre el mundo semántico, sobre las interpretaciones de las representaciones del mundo pragmático.

7. Finalmente podemos decir que el Método Científico no es ni más ni menos que la manera de hacer buena ciencia, natural o social, pura o aplicada, formal o fáctica y puede adap- tarse a campos que antes no eran científicos porque se caracterizan, al igual que la ciencia, por la búsqueda de pautas generales.

\section{NOTAS}

1 Bunge, Mario. (1997). La investigación científica. BarcelonaEspaña, Editorial Ariel.

2 Kopnin .P.V. (1966) La lógica dialéctica. México, Grijalbo.

\section{BIBLIOGRAFIA}

Bunge, M. (1997) La investigación científica. Barcelona - España, Editorial Ariel.

Bunge, M. La ciencia su método y su filosofía. Buenos Aires, Siglo XX.

Bunge. M. (1981). Epistemología. México, Ariel.

Kopnin, P.V. (1996). La lógica dialéctica. México, Grijalbo.

Moya Obeso, Alberto. La investigación científica. Trujillo-Perú, Editorial Star.

Rochabrún, Guillermo. (1977). Hacia una metodología marxista. En Revista Debates en Sociología. Pontificia Universidad Católica del Perú. Lima, № 1. 